\title{
Plasma turbulence, suprathermal ion dynamics and code validation on the basic plasma physics device TORPEX
}

\author{
I. Furno ${ }^{1} \dagger$, F. Avino ${ }^{1}$, A. Bovet ${ }^{1}$, A. Diallo ${ }^{2}$, A. Fasoli, K. Gustafson ${ }^{3}$, \\ D. Iraji ${ }^{4}$, B. Labit ${ }^{1}$, J. Loizu ${ }^{2,4}$, S. H. Müller ${ }^{1}$, G. Plyushchev ${ }^{1}$, M. Podestà ${ }^{2}$, \\ F. M. Poli ${ }^{2}$, P. Ricci ${ }^{1}$ and C. Theiler ${ }^{1}$ \\ ${ }^{1}$ Centre de Recherches en Physique des Plasmas - Ecole Polytechnique Fédérale de Lausanne, \\ CH-1015 Lausanne, Switzerland \\ ${ }^{2}$ Princeton Plasma Physics Laboratory, Princeton, NJ 08543 - USA \\ ${ }^{3}$ The Institute of Bioengineering, School of Life Sciences, Ecole Polytechnique Federale de Lausanne \\ (EPFL), 1015 Lausanne, Switzerland \\ ${ }^{4}$ Istituto di Fisica del Plasma (I.F.P.). ENEA-CNR Association, Via R. Cozzi 53, 20125-Milano, Italy
}

(Received 19 September 2014; revised 2 February 2015; accepted 3 February 2015; first published online 13 March 2015)

The TORPEX basic plasma physics device at the Center for Plasma Physics Research (CRPP) in Lausanne, Switzerland is described. In TORPEX, simple magnetized toroidal configurations, a paradigm for the tokamak scrape-off layer (SOL), as well as more complex magnetic geometries of direct relevance for fusion are produced. Plasmas of different gases are created and sustained by microwaves in the electron-cyclotron (EC) frequency range. Full diagnostic access allows for a complete characterization of plasma fluctuations and wave fields throughout the entire plasma volume, opening new avenues to validate numerical codes. We detail recent advances in the understanding of basic aspects of plasma turbulence, including its development from linearly unstable electrostatic modes, the formation of filamentary structures, or blobs, and its influence on the transport of energy, plasma bulk and suprathermal ions. We present a methodology for the validation of plasma turbulence codes, which focuses on quantitative assessment of the agreement between numerical simulations and TORPEX experimental data.

\section{Introduction}

Plasmas in nature (planet magnetospheres, stars, accretion disks, extragalactic jets, interstellar medium, etc.) and in the laboratory (industrial applications, nuclear fusion) are permeated by waves, instabilities and turbulence, which are associated with an anomalously large transport of thermal and suprathermal particles across magnetic fields. Understanding and possibly controlling instabilities and turbulence, and the resulting anomalous transport is of primordial importance for a variety of plasma systems.

In the basic plasma physics and applications group at the CRPP, waves, instabilities and turbulence studies have been initiated on the TORPEX device (Fasoli et al. 2003, 2010). The approach has been from its beginnings to advance our understanding by combining numerical simulations and basic plasma physics experiments, which offer 
full access for diagnostics and freedom in the variability of key control parameters. With a continuously improving set of diagnostic techniques, of theoretical and modeling tools, together with a detailed methodology for validating them, research on TORPEX has attained a level at which quantitative comparisons between theory and experiment can be performed. This has allowed reaching a fairly complete understanding of the linear instabilities emerging in simple magnetized toroidal configurations and of the meso-scale turbulent structures that nonlinearly develop from them. Significant advances have been obtained also in the understanding of the resulting transport of the different plasma components, in view of possible control strategies.

This article is organized as follows. In Sec. 2, we describe the main technical elements of the TORPEX device, together with its major diagnostics and the suprathermal ion system. Section 3 details the advances obtained in four major areas of research: the identification of instabilities underlying turbulence in a simple magnetized torus (SMT) and their universality aspect; the generation of blobs, their dynamics and control by the creation of convective cells; the turbulent transport of suprathermal ions; and finally, the development of a new methodology for code validation. Future opportunities for research are outlined in Sec. 4.

\section{Machine description: the TORPEX device, its diagnostics and ancillary systems}

The TORoidal Plasma EXperiment (TORPEX) is a highly flexible basic plasma physics device allowing for full diagnostic access and a variety of magnetic configurations. Plasma with densities $n_{e} \approx 10^{15}-10^{17} \mathrm{~m}^{-3}$ and temperatures $T_{e} \approx$ $5-10 \mathrm{eV}$ are produced and sustained by microwaves at $2.45 \mathrm{GHz}$ using different gases. The main TORPEX elements are reviewed in this Section.

\subsection{Vacuum vessel}

TORPEX is a toroidal device with major radius $R=1 \mathrm{~m}$ and minor radius $a=0.2 \mathrm{~m}$. A drawing of the device with the main elements is shown in Fig. 1. The circular crosssection vacuum chamber is made of stainless-steel and is divided into 12 sectors, each spanning over $30^{\circ}$. The fourfold symmetrical vessel is divided into four electrically insulated sets, of three sectors each, to limit induced eddy currents. Four sectors of the vessel, toroidally separated by $90^{\circ}$ from each other, are extractable, so that they can be easily removed to facilitate the installation of diagnostics. Spare sectors are available and can be modified for new experiments, without stopping the experimental activity. A total number of 48 port-holes provides access for diagnostics and pumping. Four turbo-pumps (nominal pumping speed of $3451 \mathrm{~s}^{-1}$ for nitrogen), backed by four primary pumps, maintain a base pressure $\lesssim 5 \times 10^{-5}$ mbar inside the vessel. Gas or mixture of different gases (Argon, Hydrogen, Deuterium, Helium, and Neon) are injected at one toroidal position; the injection rate is remotely controlled.

\subsection{Magnetic coils: simple magnetized torus (SMT) and closed field line configurations}

The TORPEX magnetic field coil system comprises a toroidal, a poloidal and an ohmic system allowing for a variety of magnetic configurations. The toroidal system (Fig. 1) consists of 28 water-cooled coils for the generation of the toroidal magnetic field. This can be varied up to $\sim 100 \mathrm{mT}$ on axis and is limited by the maximum coil current of $400 \mathrm{~A}$. The poloidal coil system includes 10 different water-cooled coils and allows for a variety of different vertical magnetic field configurations (see Müller 


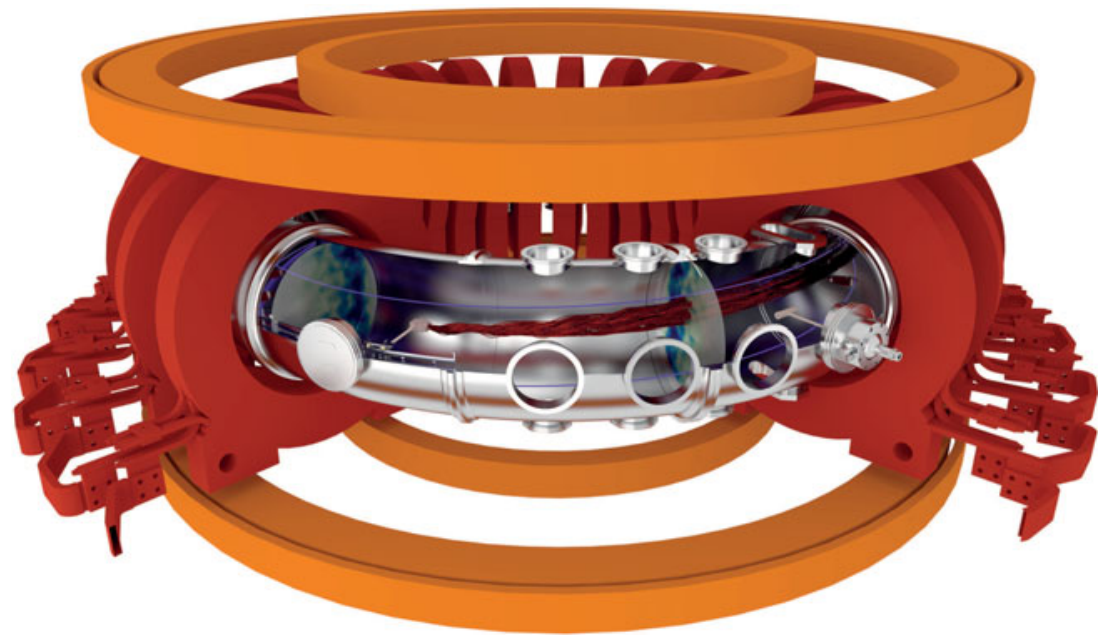

FIGURE 1. View of the TORPEX device with the main elements. A helical magnetic field line of a SMT configuration is shown in violet. The suprathermal ion source mounted on the toroidal moving system and one detector on a $2 \mathrm{D}$ positioning system are also shown. The detectors can be placed at different toroidal distance from the source. The combination of those two moving systems allows the suprathermal ion current profile to be measured in three dimensions (see Sec. 6). Examples of simulated suprathermal ion trajectories computed for $30 \mathrm{eV}$ ions are shown in red. Simulated plasma potential profiles at two toroidal positions are shown. These are obtained from numerical simulations using the GBS code (see Sec. 3.4).

et al. (2007) for details) with a maximum vertical field of $5 \mathrm{mT}$, corresponding to a maximum current of 300 A flowing into the coils. The design and arrangement of the poloidal coils are optimized to provide also a cusp field up to $100 \mathrm{mT}$ about $10 \mathrm{~cm}$ from the center of the vessel, with a magnetic null (or X-point) at the center of the cross section. The cusp field configuration has not been investigated to date. A subset of the poloidal coils can be used to induce in the vessel a loop voltage of the order of $10 \mathrm{~V}$ for approximately $30 \mathrm{~ms}$, in a tokamak-like configuration. A first proof of principle of these experiments in an ohmic configuration was obtained (Furno et al. 2006).

The combination of a toroidal magnetic field $B_{T}$ with a vertical magnetic field $B_{z}$ results in helical open field lines, terminating on the vacuum vessel. An example of magnetic field lines is shown in Fig. 1. This magnetic geometry, known as SMT, incorporates the main ingredients for drift and interchange instabilities and turbulence, namely pressure gradients, $\nabla B$ and magnetic field curvature. In SMTs, a quasiequilibrium forms characterized by a parallel plasma current canceling out the $\nabla B$ and curvature drifts (Müller et al. 2004). Although it does not possess a rotational transform of the magnetic field lines, the SMT mimics the crucial external region of magnetically confined plasmas for fusion, referred to as SOL, where magnetic field lines are open and terminate either on the vessel wall or on limiters/divertors.

To better mimic the SOL-edge magnetic geometry in tokamak, we recently developed and installed a new system that creates twisted field line configurations (Avino et al. (2014)). In Figs 2(a) and (b), the three-dimensional CAD model of the new system and a picture of the toroidal copper conductor installed in TORPEX are showed. The toroidal copper conductor ( $1 \mathrm{~cm}$ radius) is suspended inside the vacuum vessel by an electrical coaxial feed-through, Fig. 2(b), and by three vertical stainless steel wires $(1 \mathrm{~mm}$ diameter). Four horizontal supports are also used to stabilize 

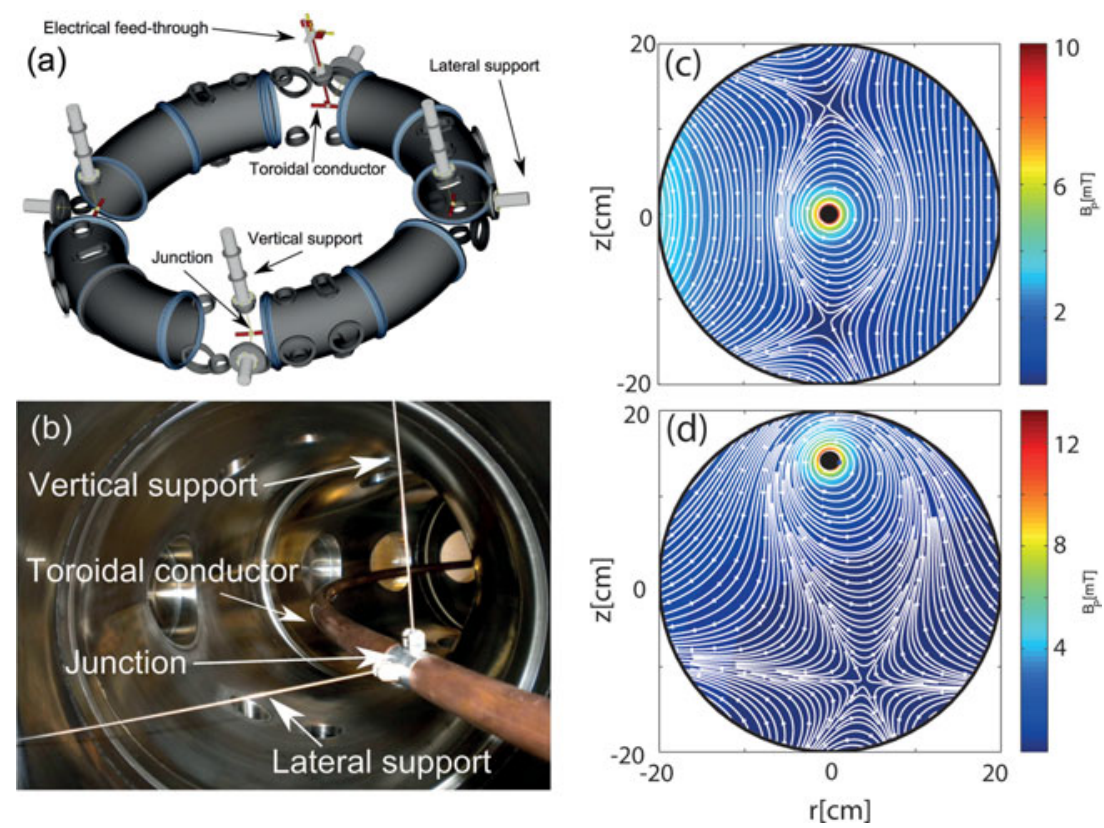

FIGURE 2. Schematic 3D view (a), and picture (b), of the in-vessel toroidal conductor with the main elements installed on TORPEX. Simulation of a (c) double-null configuration and (d) a magnetic second order null-point (magnetic snowflake) on TORPEX.

the conductor during a TORPEX discharge. This set of supports enables different vertical positions of the toroidal conductor, so that the SMT configuration can be recovered by pulling it to the top of the vacuum vessel. A dedicated external power supply drives current in the toroidal conductor up to $\sim 1 \mathrm{kA}$. The output dynamics of the power supply is limited to a maximum slew rate of $\approx 1400 \mathrm{As}^{-1}$. This allows reaching a maximum flat top current in approximately $700 \mathrm{~ms}$. For simplicity, only the feed-through is water-cooled. The flat top current duration is limited by the Ohmic heating of the conductor with almost pure radiative cooling in vacuum. This new system opens the possibility of investigating plasma fluctuations in the presence of a rotational transform in either confined regions with closed magnetic flux surfaces, or SOL regions with open field lines. Several new magnetic field configurations are possible, Figs 2(c) and (d) and Fig. 8(a), from simple plasmas limited on the low-field side (LFS) by the vessel, to more complex magnetic geometries, such as single or double-null X-points, as well as advanced divertor concepts like snowflakes (Ryutov 2007; Piras et al. 2009).

\subsection{Plasma production}

TORPEX plasmas are produced and sustained by microwaves at $2.45 \mathrm{GHz}$, corresponding to the EC range of frequencies. The choice of the frequency is based on the constraints imposed by the maximum available toroidal magnetic field, $B_{T} \approx 0.1 \mathrm{~T}$, and by the large availability of sources in this frequency range for industrial applications. The microwave system installed on TORPEX consists of a commercial microwave source and a transmission line, which connects the source to the vacuum chamber. The source can deliver up to $5 \mathrm{~kW}$ of microwave power in continuous mode, or up to $50 \mathrm{~kW}$ during $100 \mathrm{~ms}$ in pulsed mode. The microwave power can be modulated with frequencies up to $100 \mathrm{kHz}$ for a sinusoidal waveform, 
or $20 \mathrm{kHz}$ for a square waveform. The modulation amplitude is arbitrary, with a lower limit for the injected power of $200 \mathrm{~W}$. The injected and reflected power are measured on the transmission line and provide the value of the power absorbed by the plasma. For practical convenience, the microwaves are injected perpendicularly to the magnetic field from the LFS. A truncated waveguide is used as antenna, which introduces finite $k_{\perp}$ components in the injected spectrum. The ordinary (O-mode) polarization has been chosen to avoid the cut-off for the extraordinary mode at relatively low densities. A complete description of the TORPEX microwave system can be found in Podestà et al. (2007).

The plasma production mechanism associated with the injection of microwaves in the EC range of frequencies in STM plasmas was investigated in great detail (Podestà et al. 2005, 2006, 2007). The main contribution to the particle source comes from the electrons accelerated at the EC and upper-hybrid resonant layers. The electrons in the tail of the thermal distribution give only a negligible contribution to the total ionization rate. The dependence of the plasma source upon experimental parameters, such as the absorbed microwave power, the magnetic field configuration, and the neutral gas pressure, was also investigated and an expression for the spatial profile of the particle source is derived from the experimental results and a simple numerical code. These studies are of paramount importance for the code validation program described in Sec. 3.4.

\subsection{Diagnostics and ancillary systems}

The relatively low temperatures and densities of TORPEX plasmas allow using internal probes for a full reconstruction of plasma profiles and turbulent structures across the entire device. TORPEX possesses an extensive set of diagnostics, comprising approximately 200 probes, together with a fast optical imaging system. These are briefly described in this section together with a specialized setup to inject and detect suprathermal ions.

\subsubsection{Electrostatic probes.}

Measurements of time-averaged plasma parameters and electrostatic fluctuations are performed using combinations of arrays of Langmuir probes (LP) in single, double, and triple configuration (Theiler et al. 2011b), and Mach probes (Labit et al. 2011). The LPs can be operated in ion saturation, floating potential, or swept mode and their signals are acquired at a rate of $250 \mathrm{kHz}$. The LPs are disposed at different toroidal locations and are installed on remotely-controlled $2 D$ positioning systems, which allow covering almost the entire plasma poloidal cross-section by moving the probes in between discharges.

An array of 85 LPs at one toroidal location, the HEXagonal Turbulence Imaging Probe (HEXTIP), allows characterizing fluctuations and turbulent structures in terms of wavelength, coherence lengths, frequency, speed, and statistical properties, in a single plasma discharge (Müller et al. 2006). The original HEXTIP design with 86 probes was recently modified to be compatible with the new toroidal conductor system. HEXTIP covers the entire poloidal cross-section with a spatial resolution of $3.5 \mathrm{~cm}$ and constitutes the workhorse for a high temporal resolution $(250 \mathrm{kHz})$ real space imaging of the ion saturation current and floating potential dynamical evolution. HEXTIP allows identifying observables related to turbulent structures, such as size, mass, shape, and trajectories from two-dimensional plasma imaging data. The probability distributions of these observables from measurements of many turbulent realizations, can then be used to characterize the nature of turbulence and 
the related transport. HEXTIP ion saturation data illustrating blob dynamics are shown in Figs 5(a)-(c). An example of structure analysis is presented in Fig. 8 of Sec. 4. A new version of the HEXTIP array with improved (and variable) spatial resolution has recently been developed. Two new systems are under construction and will be installed in TORPEX at different toroidal locations, thus extending to $3 D$ the measurements available with the previous HEXTIP.

Special arrangements of single and multiple single-sided LPs are used to measure plasma currents collected on a poloidal limiter, in particular for blob studies (Furno et al. 2011a). The simplest version of these probes consists of a conductor plate (usually made out of tungsten) with a collecting area $A_{L P}$, which is exposed to the plasma only from one side a few $\mathrm{cm}$ in front of the limiter. The plate is oriented perpendicularly to the magnetic field and is kept at the limiter potential, such that the current to the limiter, $I_{0}$, is now measured by the probe. The current density is then computed as $J_{\|}=I_{0} / A_{L P}$. A good agreement is found between single-sided probe and magnetic probe measurements (Furno et al. 2011b).

\subsubsection{Magnetic probes.}

Magnetic and current probes complement electrostatic probes and provide $3 D$ fluctuating components of the magnetic field with $\mathrm{nT}$ resolution. A specially designed magnetic probe, developed in collaboration with Consorzio RFX in Padova (IT), consists of an L-shaped array of 3 miniaturized 3-axial pick up coils $(3.5 \mathrm{~cm}$ spaced, effective area of $2.3 \times 10^{-3} \mathrm{~m}^{2}$ ) (Furno et al. 2011a,b). The probe is installed on a 2D movable system and allows mapping of the magnetic field and current density fluctuations associated with turbulent structures (Furno et al. 2011a,b).

Together with magnetic probes, direct measurements of the current density are obtained using a dedicated miniaturized current probe diagnostic (Fasoli et al. 2013). The probe consists of a Rogowski coil wound around a ferrite core to amplify the magnetic flux. Electrical and thermal insulation from the plasma is obtained by placing the detecting coil inside a vacuum safe cylindrical housing, machined as two halves of a container. The toroidal detecting coil consists of a single layer helical winding with an inside return loop to cancel the net single turn formed by the solenoid windings. The probe is operated in current mode, which provides a direct measurement of the total current flowing inside the detection area. Preliminary measurements of blob current structures with this probe are currently ongoing.

\subsubsection{Fast framing camera and intensifier.}

Electrostatic probes are complemented by a fast optical imaging system (Iraji et al. 2008), purchased in collaboration with ENEA-CNR-Milan. The system comprises a Photron Ultima APS-RX framing camera and a Hamamatsu image intensifier. The system monitors visible light fluctuations, up to $250 \mathrm{kframes} \mathrm{s}^{-1}$. Such system allowing fully non-perturbative reconstruction of plasma dynamics at different scales (Iraji et al. 2011), with a spatial resolution as high as a few $\mathrm{mm}$. The fast framing camera is equipped with a 10 Bit CMOS monochrome sensor with $1024 \times 1024$ pixels (17 $\mu \mathrm{m}$ size), which provides extreme light sensitivity up to 6400 ASA in the visible range. At the full chip resolution, the framing rate can be varied in the range 50 fps $-3 \mathrm{kfps}$, while at reduced chip size (down to $16 \times 28$ pixels), the framing rate can be increased up to $250 \mathrm{kfps}$ thus allowing fluctuation and turbulence measurements of visible light emission. The camera is equipped with an on-board 2.6 GB RAM memory, permitting the acquisition of 1s of data at full chip size and 3 fps and $4.2 \mathrm{~s}$ of data at maximum frame rate. A gas puff imaging (GPI) system is also available, 
(a)

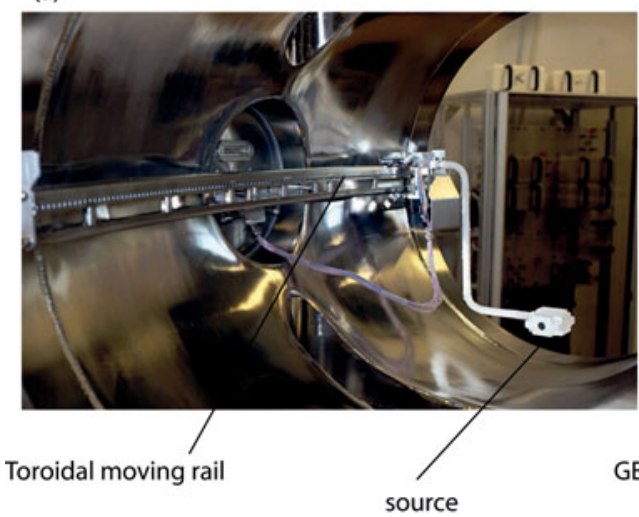

(b)

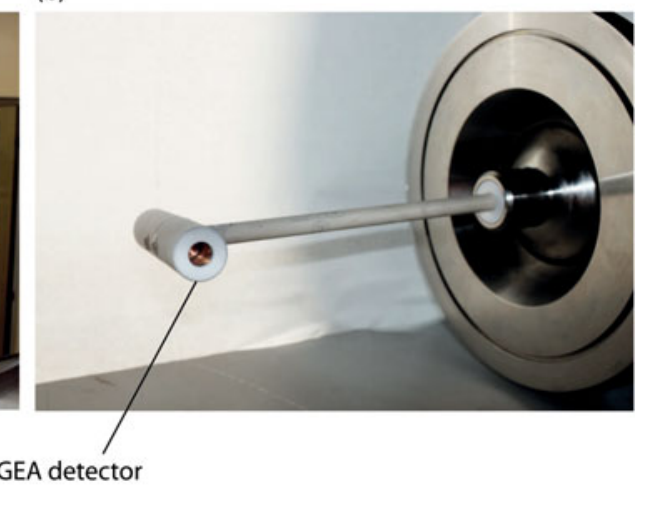

FiguRE 3. Experimental setup for suprathermal ion studies: (a) suprathermal ion source installed on a toroidal moving rail inside TORPEX and (b) gridded energy analyzer installed on a $2 D$ poloidal moving system.

including a fast piezoelectric valve and a ceramic tube with a $100 \mu \mathrm{m}$ nozzle. The use of the GPI system results in a five-fold improvement of the light collection level, which allows acquiring at higher acquisition frequencies, up to $50 \mathrm{kframes}^{-1}$ (Iraji et al. 2010).

\subsubsection{Fast ion source and detectors.}

A miniaturized $\mathrm{Li}^{+}$ion source was developed in collaboration with the University of California at Irvine, where the source was used to perform suprathermal ion studies on the LAPD experiment (Heidbrink et al. 2012). A picture of the source installed inside the TORPEX vessel is shown in Fig. 3(a). The source consists of a Li-doped thermionic aluminum-silicate emitter producing $\mathrm{Li}^{+}$ion currents up to $10 \mu \mathrm{A}$ (Plyushchev et al. 2006). The emitter is encased into a vacuum and plasma safe boron nitride casing, which includes a two-grid system to accelerate the ions. A screen grid is grounded to minimize perturbations, while the voltage between the emitter and the accelerating grid is modulated. The accelerating grid is powered by a high voltage DC-power supply. Synchronous detection of the ion beam is used to increase the signal-to-noise ratio by modulating the emitter bias voltage at a given frequency $(\approx 1 \mathrm{kHz}$ ) (Bovet et al. 2012). Varying the voltage between the accelerating and the screen grids allows focusing of the fast ion beam. $\mathrm{Li}^{+}$ions with energies in the range $100 \mathrm{eV}-1 \mathrm{keV}$ can be produced. The source is mounted on a motorized rail system and can be continuously moved over a toroidal distance of about $50 \mathrm{~cm}$.

Ion energy and current density profiles are measured using two identical gridded energy analyzers (GEAs), Fig. 3(b), which face opposite directions for background noise subtraction. Each detector has small dimensions relative to the plasma size (15 $\mathrm{mm}$ in diameter, $70 \mathrm{~mm}$ in length, and in inlet diameter of $8 \mathrm{~mm}$ ), and is able to measure fast ion currents as small as $0.1 \mu \mathrm{A}$. The GEA detector is installed on a two-dimensional moving system, which allows reconstruction of the ion current density profile with a spatial precision of $2 \mathrm{~mm}$ over almost the entire poloidal crosssection. The use of the fast ion source and detectors allows measurements of the time-averaged three-dimensional profile of the suprathermal ion beam as it interacts with the plasma turbulence, as discussed in Sec. 3.3. 
(a)

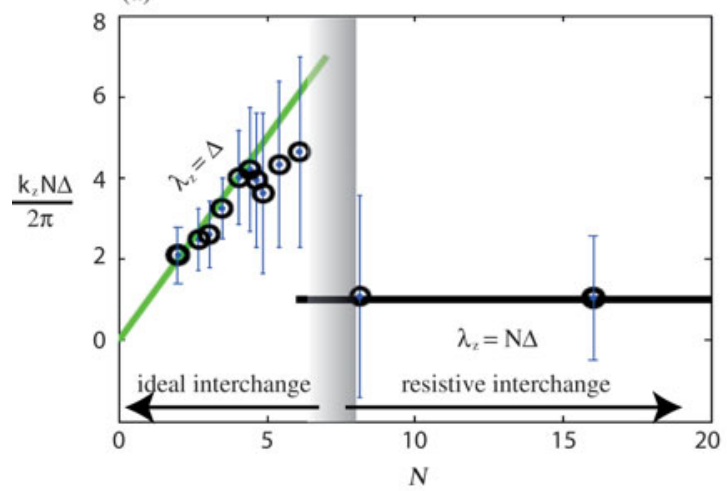

(b)

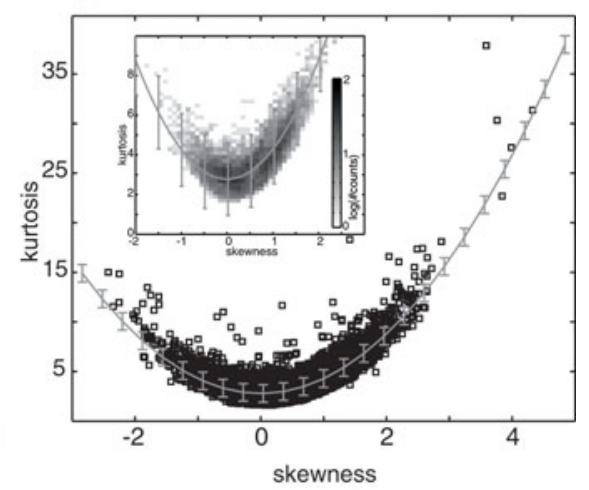

FIGURE 4. (a) Identification of the nature of the SMT instabilities. (a) Vertical wave numbers of the dominant instabilities as a function of the total number of field line turns $N$. A transition from a regime dominated by ideal interchange modes to one dominated by resistive interchange mode is clearly visible. (b) Kurtosis versus skewness computed for the $\sim 10000$ ion saturation current signals. The fitting second order polynomial (solid grey line) is also plotted. Inset: Joint probability of $K$ and $S$ for the experimental signals.

\section{Major research questions}

This section reviews the waves, instabilities, and turbulence investigations on TORPEX as follows. The characterization of the basic plasma properties and the identification of the waves and instabilities underlying turbulence is detailed in Sec. 3.1. The mechanisms for the generation of blobs, their dynamics and the development of active and passive methods to control them are discussed in Sec. 3.2. The influence of blobs and coherent modes on the transport of suprathermal ions is presented in Sec. 3.3. Finally, the validation methodology is outlined in Sec. 3.4.

\subsection{Drift, interchange modes and universality of turbulent fuctuations}

A first and essential step in the understanding of plasma turbulence is the determination of the nature of the underlying instabilities. An extensive experimental effort has been dedicated to this investigation on TORPEX in the SMT configuration, based upon detailed measurements of the wave dispersion relation both along and across the magnetic field (Poli et al. 2006, 2007, 2008). The dominant mode activity is localized in the unfavorable curvature region, i.e. on the plasma LFS. A clear transition is visible in Fig. 4(a) from a regime dominated by instabilities with finite parallel wave number to one dominated by ideal interchange instabilities, with zero parallel wave number. This transition is controlled by pitch of the field lines, which is expressed in terms of $N$, the total number of field line turns from the bottom to the top of the vessel. For $N \lesssim 7$, only ideal interchange instabilities are observed.

Following these findings, three-dimensional global fluid simulations have been run and three turbulence regimes have been found: an ideal interchange mode regime, a previously undiscovered resistive interchange mode regime, and a drift-wave regime. As the pitch of the field lines is decreased, the simulations exhibit a transition from the first regime to the second, while the third, the drift-wave regime, appears to be accessible only for very low collisionality values. The interpretation of the underlying instability as either resistive or ideal interchange has been quantitatively confirmed by the experiments. The onset of $k_{\|}=0$ fluctuations is observed for sufficiently large $N$. The existence of a critical pressure gradient for the stabilization of the 
ideal interchange modes was predicted theoretically and demonstrated experimentally (Federspiel et al. 2009). Excitation of drift-interchange waves using an external antenna was demonstrated (Diallo et al. 2007).

A significant effort was dedicated to explaining the existence of universal aspects in the statistical description of plasma turbulence (Labit et al. 2007a,b). The question of universality has been addressed by investigating over a broad range of experimental conditions a large number $(\sim 10000)$ of probability density functions (PDFs) of ion saturation current fluctuations. These are obtained across the whole plasma cross-section by binning the ion saturation current measurement from each tip of HEXTIP. Without an a priori idea on the PDF shape, a good compromise between resolution in the binned quantity and statistics is given by choosing the number of bins $N_{\text {bin }}=O\left(\sqrt{N_{p t s}}\right)\left(N_{p t s}\right.$ is the number of points in the time series). A unique and quadratic polynomial relationship was discovered, shown in Fig. 4(b), which links the experimental skewness $S$ (third moment of the PDF) and the kurtosis $K$ (fourth order moment of the PDF): $K=(1.502 \pm 0.015) \times S^{2}+(2.784 \pm 0.019)$. This relation rules out all PDF families that cannot provide such a quadratic polynomial together with these coefficients. As a consequence, a second universal character of interchange turbulence is identified: all the PDFs of the density fluctuations at all locations can be described by the same analytical distribution. This is a special case of the general beta distribution, akin to the form of the gamma distribution, depending on the sign of the signal skewness. Fluctuations in the frequency range of interchange turbulence are found to be essential to recover this beta distribution (Labit et al. 2007b).

These findings triggered a number of theoretical investigations to explain the observed universality of fluctuations including the skewness-kurtosis relation. A nonlinear Langevin equation for plasma turbulence, generalized to include linear wave propagation, was proposed (Krommes et al. 2011). Theories based on stochastic models and equations seem to be successful in recovering the KS relationship and the gamma distribution (Sandberg et al. 2012; Garcia et al. 2012; Mekkaoui, 2013). Recently, attempts have been performed to connect the coefficients of the skewnesskurtosis relation to geometrical properties of the propagating blobs (Guszejnov et al. 2012a).

An extensive experimental program has been dedicated to identifying and quantifying the different contributions to the particle cross-field flux (Podestà et al. 2008). The particle fluxes associated with the creation of the quasi-equilibrium are compared with those related to fluctuating density and potential, both locally and in conjunction with macroscopic fluctuating structures. To this aim, a modified conditional sampling technique has been developed which allows simultaneous measurements of time-resolved, two dimensional profiles of electron density, and temperature and plasma potential (Furno et al. 2008a). These measurements are complemented by direct observation of turbulent structures and related particle flux as obtained by a spatio-temporal pattern recognition method, which was previously developed on the TORPEX device (Müller et al. 2006).

\subsection{Blob physics}

In many natural and laboratory systems, particles, energy, and momentum are efficiently transported across the magnetic field by filamentary plasma structures, in which temperature and density are enhanced with respect to the background plasma. These structures, usually referred to as blobs or filaments, extend much further along the magnetic field than perpendicularly to it. Plasma filaments are observed in the solar photosphere (Otsuji et al. 2007) and in the F-layer of the ionosphere (Park 
et al. 2010), where they are aligned with the Earth magnetic field and may disturb L-band GPS communications during their propagation. In the laboratory, virtually all confinement devices have shown the presence of plasma blobs (see review D'Ippolito et al. (2011)) irrespectively of their magnetic field configuration. They are observed to dominate the transport across the plasma edge of fusion devices, which may lead to serious wall erosion, impurity, and recycling problems in future fusion reactors. A large experimental and theoretical effort has been devoted on TORPEX to investigate the origin of filaments, their dynamics and methods to control them. The major results are summarized in this section.

The mechanism for blob formation in scenarios dominated by $2 D$ ideal interchange turbulence was clearly identified (Müller et al. 2008; Theiler et al. 2008; Furno et al. 2008b; Müller et al. 2009). Blobs are observed to form from radially extending positive crests of the interchange waves that are sheared off by the $\mathbf{E} \times \mathbf{B}$ flow. The radial elongation of the wave stems from an increase of the radial pressure gradient (Diallo et al. 2008; Theiler et al. 2008; Furno et al. 2008b). This mechanism is illustrated in Figs 5 (a)-(c) using 2D profiles of ion saturation current from HEXTIP (Müller et al. 2006). In this particular set of experiments, a poloidal steel limiter is inserted in the outer half of the cross section, where blobs propagate (Theiler et al. 2009). The elongation of the wave crest is shown in Fig. 5(a), while the radial propagation of the blob is clearly visible in Figs 5(b) and (c). Having elucidated the mechanism for blob formation, we have then focused on the dynamics of blob propagation. The goal was to identify and quantify the different effects that determine the blob crossfield velocity and, ultimately, to develop methods to influence blob motion, hence cross-field transport.

From the theory point of view (Krasheninnikov 2001), the blob motion results from $\nabla B$ and curvature, which cause blob polarization and a corresponding $\mathbf{E} \times \mathbf{B}$ convection. To satisfy charge conservation, $\nabla \cdot \mathbf{J}=0$, both perpendicular currents inside the blob (i.e. ion polarization currents and/or currents due to ion-neutral collisions) and currents parallel to the magnetic field into the limiter arise, which damp the electrical polarization of the blob and, thus, its radial velocity. Using dimensionless quantities $\widetilde{a}=a / a^{*}$ and $\widetilde{v}=v / v^{*}$, where $a$ and $v$ are blob vertical size and radial speed respectively, and $a^{*}=\left(4 L^{2} / \rho_{s} R\right)^{1 / 5} \rho_{s}$ and $v^{*}=\left(2 L \rho_{s}^{2} / R^{3}\right)^{1 / 5} c_{s}$, we proposed an analytical expression for $\widetilde{v}$, shown in Fig. 5(d) with continuous lines, which takes all of the above effects into account

$$
\widetilde{v}=\frac{\sqrt{2 \widetilde{a}}}{1+\sqrt{2} \widetilde{a}^{5 / 2}+\widetilde{\eta} \sqrt{a}} \frac{\delta n}{n} .
$$

Here, $\widetilde{\eta}$ represents a normalized ion-neutral collision frequency (see Theiler et al. (2009) for the definition). The three terms in the denominator represent, in the order, the damping of the blob velocity due to inertia, parallel currents to the sheath, and ion-neutral collision, while $\delta n / n$ describes the slowing down by a finite background density (Theiler et al. 2009). The relative importance of these terms depends on the normalized blob vertical size $\tilde{a}$. The term corresponding to parallel currents becomes dominant for $\widetilde{a}^{5 / 2} \gg 1, \widetilde{\eta} \sqrt{a}$. In this case, the scaling $\widetilde{v}=1 / \widetilde{a}^{2}$ of Krasheninnikov (2001) is retrieved. In the limit where sheath losses and ion-neutral collisions are negligible, for $\widetilde{a}^{5 / 2}, \widetilde{\eta} \sqrt{\tilde{a}} \ll 1$, the scaling $\widetilde{v}=\sqrt{2 \widetilde{a}}$ of Garcia et al. (2005) and Myra and D'Ippolito (2005) is retrieved. Ion-neutral collisions become the dominant damping term when $\widetilde{\eta} \gg 1 / \sqrt{\tilde{a}}, \widetilde{a}^{2}$. In this limit we retrieve the scaling presented in Katz et al. (2008). 


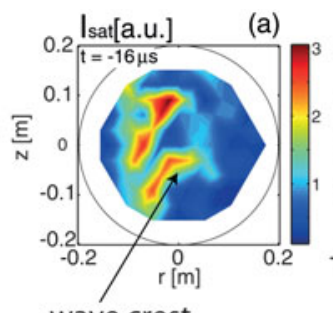

(b)
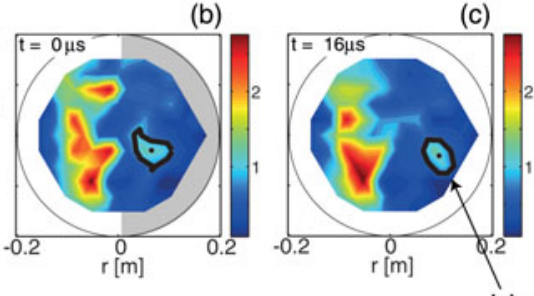

wave crest
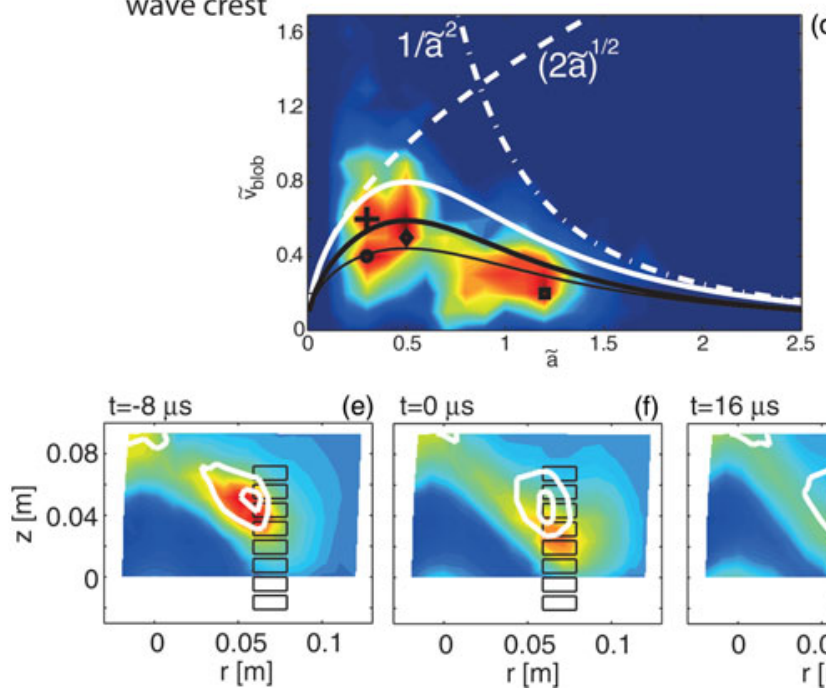

$t=0 \mu \mathrm{s}$

(f) $t=16 \mu \mathrm{s}$

(d)

blob
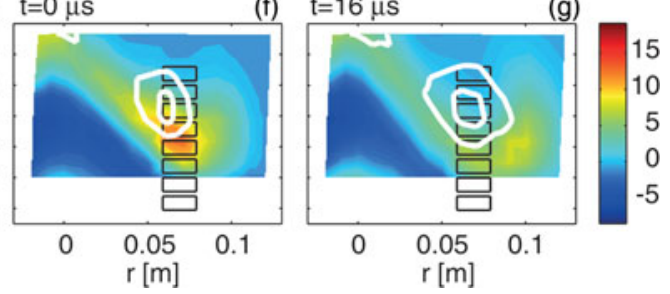

FIGURE 5. Blob physics: (a)-(c) 2D profiles of ion saturation current from HEXTIP, showing the generation of an individual blob from a wave crest of an interchange mode. For the present experiments, a steel limiter, schematically shown in (b), is inserted in the outer half of the cross section, i.e. $r>0$, where blobs propagate; (d) Scaling law for $\widetilde{v}_{b l o b}$ versus $\widetilde{a}$. The joint probability is shown for different gases. For clarity, the distribution for $H_{2}(\square)$ and $\mathrm{He}(\diamond)$ are normalized to 1 , the ones of $\mathrm{Ne}(+)$ and $\mathrm{Ar}(\bigcirc)$ to 0.5 . The symbols indicate the peak of each distribution. White line, thick black $(\delta n / n=1, \eta=0)$ and thin black lines correspond to $\delta n / n=1, \widetilde{\eta}=0$, $\delta n / n=0.73, \tilde{\eta}=0$, and $\delta n / n=0.73, \widetilde{\eta}=0.6$, respectively. (e) $-(\mathrm{g})$ Blob control by convective cells. Conditionally averaged blob propagation of ion saturation fluctuations for the case where a vertical stripe of electrodes is biased to $+40 \mathrm{~V}$ (color plots). For comparison, the white contours indicate the results of the same analysis when all the electrodes are grounded.

The scaling provided by (3.1) is compared in Fig. 5(d) with experimental blob speedversus-size joint probabilities over a wide range of the key dimensionless parameters in TORPEX data, vastly exceeding tokamak SOL databases, obtained using different gases. The scaling is in good agreement with the experimental data, showing that two different regimes of blob motion are achieved in TORPEX, in which parallel currents to the sheath do or do not significantly damp charge separation, and thus blob radial velocity. Recently the effect of the neutral gas density upon the blob velocity was also investigated (Furno et al. 2014). The existence of these two regimes is also confirmed by time-resolved two-dimensional measurements of the field-aligned current density $J$ associated with blobs (Furno et al. 2011a). The profile of $J_{\|}$exhibits an asymmetric dipolar structure, which originates from the polarization of the blob and is consistent with sheath boundary conditions and numerical simulations (Furno et al. 2011b).

Based on our understanding of the physics governing blob motion, we started to develop and test different methods to control the blob dynamics. As a first attempt, 
limiter configurations with varying angles between field lines and the conducting surface of the limiter were explored but proven unsuccessful (Theiler et al. 2011a), while varying the connection length or the neutral gas pressure showed the expected effects.

Another method, aimed at inducing radial flows and convective cells, is based on the use of biased plates. This technique that could potentially provide a tool to alleviate the divertor heat flux issue in tokamaks, has been successfully tested in TORPEX. A set of 24 electrodes installed on a metal limiter allows modifying the plasma potential and thus the cross-field flows in a controlled way (Theiler et al. 2012,b). Figures 5(e)(g) presents the effect on blobs, when a bias of $+40 \mathrm{~V}$ is applied to the set of electrodes indicated by black rectangles. Conditional average sampling is applied to obtain the ensemble average blob propagation over the $2 D$ poloidal plane. In the same figure, the white contours depict the conditional sampled blob in the case without biasing (i.e. all electrodes are grounded). While early in time the average blob evolution is very similar with and without biasing, later on, as anticipated, the blob is swept strongly downwards due to the applied bias. The blob vertical velocity in the time window [ $-8 \mu \mathrm{s}, 8 \mu \mathrm{s}]$ is modified from $\sim 700$ to $\sim 2100 \mathrm{~m} \mathrm{~s}^{-1}$ due to the biasing. Depending on the biasing scheme, radial blob velocities can also be varied significantly. Biasing also results in significant modifications of the time-averaged profiles, including the electron density profile, although the magnitude of the achievable potential variations is well below the potential applied to the electrodes. Analytical estimates, based on the drift-reduced Braginskii ordering valid in TORPEX, have been performed to evaluate the magnitude of the cross-field currents that presumably underlie the reduction of the potential variations with respect to the applied bias. These estimates show that the high level of cross-field currents necessary to explain the experimental observations cannot be accounted for by ion polarization currents, diamagnetic currents, or currents due to ion/electron-neutral collisions and gyro-viscous effects (Theiler et al. 2012b; Fasoli et al. 2013).

\subsection{Suprathermal ion physics}

A wide variety of plasmas in nature and in the laboratory are characterized by the presence of suprathermal ions (Perrone et al. 2013), i.e. ions with energies much larger than the background plasma. These can be generated by coherent or turbulent acceleration mechanisms or by shocks (Reames 1999; Perri and Zimbardo 2012), or directly by external sources (neutral beams and/or EC resonance heating (ECRH and current drive (CD), (Goodman et al. 2003; Henderson et al. 2003)) or nuclear reactions, as in the case of magnetic fusion devices. Common to all of these systems is the question of how plasma turbulence influences suprathermal particles and, in turn, how suprathermal particles influence turbulence. This problem is receiving much attention today. For example, in fusion research, a good confinement of the slowing down suprathermal $\alpha$-particles and the NBI- and/or ECRH/ECCD-generated fast ions is a crucial requirement for the burning plasma regime, as they will be responsible for a dominant fraction of plasma heating. Understanding the transport of suprathermal ions is therefore of fundamental importance. In TORPEX, we started theoretical and experimental investigations of suprathermal ion-turbulence interaction in the SMT configuration using extensive sets of $3 D$ data, together with numerical simulations of suprathermal ion tracers in fluid turbulent fields. The major results are detailed in this Section.

The experiments are conducted in a plasma scenario dominated by an ideal interchange mode, characterized by $k_{\|} \approx 0$ and localized around the position of 
maximum pressure gradient. The time-averaged electron density at the injection location is $n_{e}=5 \times 10^{15} \mathrm{~m}^{-3}$ and the standard deviation of the floating potential time series, indicating the level of fluctuations, is $\approx 1 \mathrm{~V}$. These plasmas, similarly to those detailed in Sec. 3.2, are characterized by the presence of a region on the LFS, where blobs are observed to propagate radially outward resulting in intermittent transport of particles, heat, momentum and current. Suprathermal ions are injected horizontally in the blob region with energy of $70 \mathrm{eV}$. With the present plasma parameters, the relevant collision frequencies of the suprathermal $\mathrm{Li}^{+}$ions with background thermal ions are $v_{s, i} \approx 16 \mathrm{~s}^{-1}$ and with neutrals $v_{s, n} \approx 6.2 \times 10^{3} \mathrm{~s}^{-1}$, respectively. As these frequencies are typically much smaller than the gyrofrequency $\approx 188 \times 10^{3} \mathrm{~s}^{-1}$ for $\mathrm{Li}^{+}$ions in TORPEX and the ideal interchange growth rate is $\approx 8 \times 10^{4} \mathrm{~s}^{-1}$ (Furno et al. 2008a), the suprathermal ions are magnetized. For reference, the respective collision frequencies for the thermal ions are $v_{i, i} \approx 7 \times 10^{3} \mathrm{~s}^{-1}$ and with neutrals $v_{i, n} \approx 5 \times 10^{4} \mathrm{~s}^{-1}$.

By toroidally moving the source over a distance of $\approx 50 \mathrm{~cm}$ in between discharges, and the GEAs over a distance of $\sim 2.2 \mathrm{~m}, 3 D$ measurements of the suprathermal ion beam profiles are obtained. Figure 6(a) shows suprathermal ion current poloidal profiles at three toroidal distances from the source. The radial standard deviation of the suprathermal ion current profiles is shown as a function of the toroidal distance in Fig. 6(b). In the absence of a plasma, the motion of suprathermal ions in TORPEX is a combination of the vertical drift due to $\nabla B$ and curvature of the magnetic field and the gyromotion around the magnetic field lines at the cyclotron frequency. This latter results in beam width oscillations both in space and time as shown in Figs 6(d) and (e). The same figure also reveals that on top of the unperturbed motion, a broadening due to the interaction with the plasma turbulence is present.

To interpret the experimental data, we follow ion tracer trajectories using the full Lorentz force, with turbulent fields specified by a numerical model of drift-reduced Braginskii equations in two dimensions (Bovet et al. 2012, 2013). This model is implemented in the Global Braginskii Solver (GBS) code (Ricci et al. 2012) and was previously validated to describe interchange turbulence in TORPEX, as described in Sec. 3.4. Comparisons with the simulations are possible using a synthetic diagnostic, allowing the poloidal cross-section of the fast ion current density to be represented. Using the simulated turbulent electric field, tracer $\mathrm{Li}^{+}$ion trajectories are computed. Source parameters are based on measurements done without magnetic fields; $1.6 \times 10^{5}$ particles are launched with initial parameters modeled with Gaussian distributions, with a mean energy of $70 \mathrm{eV}$ and a relative standard deviation of $10 \%$. A synthetic diagnostic, representing the phase space acceptance of the detector, computes $3 D$ profiles of the fast ion current density. The comparison between the measured and simulated evolution of the beam width as a function of the toroidal distance is shown in Fig. 6(d). The radial beam width is computed as the radial standard deviation of the ion current profiles for the $70 \mathrm{eV}$ ions. A good agreement between experiment and simulation is apparent. In the same Figure, the same simulated quantities are also computed for an ion beam with $30 \mathrm{eV}$ of energy. As the distance from the source is increased, the radial width of the $30 \mathrm{eV}$ ion beam grows much faster than that of the $70 \mathrm{eV}$ ions, indicating a stronger interaction with the turbulent fields.

To fully characterize suprathermal ion transport, theoretical activities are conducted in parallel. Transport of tracers in plasmas can be characterized by the mean-squared displacement (variance of displacement) of an ensemble of individuals, which generally exhibits a power-law behavior in time, i.e. $\left\langle\left(r(t)-r_{0}\right)^{2}\right\rangle \sim t^{\gamma}$, where $r(t)$ represents the positions of individuals. When $\gamma>1$ or $\gamma<1$, the transport is called super-diffusive 

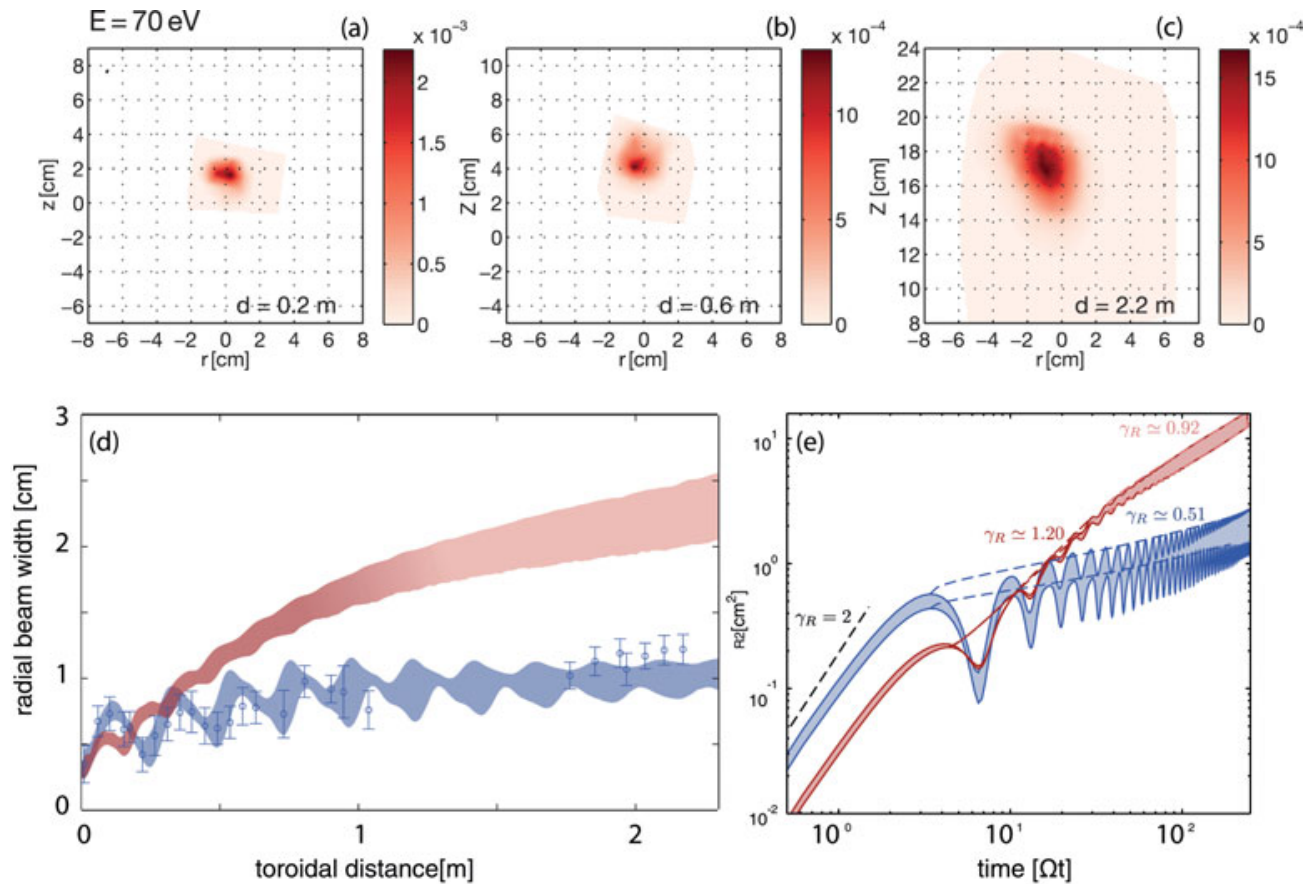

FiguRE 6. Suprathermal ion physics. (a)-(c) Poloidal suprathermal ion current profiles at different toroidal distances for $70 \mathrm{eV}$ ions. (d) Radial width of suprathermal ion current profiles as a function of the toroidal distance. Blue circles represent experimental measurements for 70 $\mathrm{eV}$ ions. Continuous bands are drawn from a synthetic diagnostic of numerical simulations for $30 \mathrm{eV}$ (red) and $70 \mathrm{eV}$ (blue) ions. (e) Variance of the ion radial positions as a function of time (normalized to the ions gyro-period) obtained from the numerical simulations reproducing the experimental data in Fig. 3, for ions at $70 \mathrm{eV}$ (blue). The beam width oscillations in space (d) and time (e) are due to the gyromotion of the ions.

or sub-diffusive, respectively. Classical diffusion is retrieved for the special case of $\gamma=1$. To compute the value of the radial transport exponent, $\gamma_{R}$, the evolution of the variance of the ion radial displacements as a function of time is computed from the numerical simulations reproducing the experimental conditions in Fig. 6. The results are shown in Fig. 6(e). After a short ballistic phase lasting approximately one gyro-period, during which the ions have not yet interacted with the plasma, the ions enter a second phase exhibiting different transport regimes depending on the energy of the ions and the character of the turbulence. A numerical study showed that in this interaction phase the transport can vary from a sub-diffusive to super-diffusive regime depending on two parameters that determine the relative sizes of the ion orbits and the turbulent structures (Gustafson et al. 2012a,b) the injection energy normalized to the electron temperature, $E / T_{e}$, and the normalized amplitude fluctuations, $\Phi / T_{e}$. Fitting the temporal evolution of Fig. 6(e) to power laws provide the values of the transport exponents in the different phases. In the interaction phase, an exponent $\gamma_{R}=0.51 \pm 0.01$ is found for ions of $70 \mathrm{eV}$ and $\gamma_{R}=1.20 \pm 0.04$ for ions of $30 \mathrm{eV}$, indicating that the transport varies from sub-diffusive to super-diffusive as the energy of the ions is decreased. For ions of $30 \mathrm{eV}$, after the super-diffusive phase, a phase where the transport is close to diffusive $\left(\gamma_{R}=0.92 \pm 0.04\right)$ follows. This is visible in Fig. 6(e) after approximately six gyro-periods, corresponding to $\approx 1 \mathrm{~m}$ in Fig. 6(d). 
This quasi-diffusive phase appears when the suprathermal ion beam starts covering plasma regions with different levels of fluctuation amplitude.

The observed transport of suprathermal ions is consistent with a non-diffusive transport theory that successfully describes the simulations Gustafson et al. (2012a,b). Numerical simulations agreeing with the present measurements determine the radial transport exponents $\gamma_{R}$ and reveal the basic physical mechanisms leading to super- or sub-diffusive behavior. Suprathermal ions with lower energy follow Lévy-type superdiffusive transport trajectories as they are transported by the turbulent structures (Gustafson and Ricci 2012). For larger energies, the size of the ion orbits becomes comparable to the characteristic dimension of the turbulent structures. In this case, suprathermal ions effectively average the turbulent $\mathbf{E} \times \mathbf{B}$ drifts as they perform their gyromotion, which results in anti-correlated sub-diffusive transport. On a macroscopic level, a generalization of the classical model of diffusion, the so-called fractional Lévy motion, which encompasses power-law (Lévy) statistics for the displacements and correlated temporal increments, leads to non-diffusive dynamics such as that observed in the experiment (Bovet et al. 2014). This work provides a proof-of-principle that the transport of suprathermal ions in turbulent plasmas can exhibit various non-diffusive regimes. The importance of the parameters regulating the orbit averaging effect of turbulence, in particular $E / T_{e}$, is confirmed. This also suggests that modifying the spatial size of the turbulent structures or the level of turbulence fluctuations in the plasma, for example via flow shear control (Schaffner et al. 2012), may, in turn, influence/control suprathermal ion transport.

\subsection{Code validation}

Validating numerical codes is of upmost importance to assess the maturity of the understanding of plasma dynamics and the predictive capabilities of simulations (Terry et al. 2008; Greenwaldet al. 2010). A validation project can be thought as a four-step procedure: (i) the model needs to be qualified by establishing the applicability of the model hypotheses for the simulated physical phenomenon; (ii) verification of the code is necessary to prove that the code solves correctly the model equations; (iii) simulations and experiments have to be compared and analyzed using the same techniques, considering a number of observables, i.e. physical quantities, which are common to the experimental measurements and simulation results (Ricci et al. 2009); finally (iv) the agreement between simulations and experiments needs to be quantified using an appropriate composite metric, $\chi$. Model qualification and code verification (point (i) and (ii) of the validation guidelines) have been routinely considered in plasma physics and the related methodology has been formulated in considerable detail (Riva et al. 2014). However, only recently has the plasma physics community approached a rigorous methodology for establishing the validation observables and the comparison metric. Combining the state-of-the-art GBS code (Ricci et al. 2012) together with experimental data from TORPEX, we have carried out a validation project, of which the main highlights are illustrated below.

An essential aspect of the validation project on TORPEX is the identification of a primacy hierarchy for observables that measures how stringent each observable is for the comparison. Such hierarchy quantifies how directly are the variables extracted from the fundamental data, e.g. how the measurements are combined together and what assumptions are used in the models employed to form the observables. At the lowest levels in the primacy hierarchy are the observables that require the smallest number of model assumptions and correspond to the most direct measurements, for example ion saturation current measurements in the experiments. Vice-versa, at the 
highest levels, we place quantities that require the highest number of assumptions and are obtained by combining different measurements, such as the turbulent particle flux. We also point out that the same techniques must be used to analyze experimental and simulation data, in order to directly compare the experimental and simulation values of each observable.

First, we start by computing the level of agreement between each individual $j$ th observable denoted by $e_{j}$ and $s_{j}$, as coming from the experimental measurement or the simulation results, respectively. We define a normalized distance $d_{j}$ between experiments and simulations with respect to the uncertainty related to these quantities as follows:

$$
d_{j}=\sqrt{\frac{1}{N_{j}} \sum_{i=1}^{N_{j}} \frac{\left(e_{j, i}-s_{j, i}\right)^{2}}{\Delta e_{j, i}^{2}+\Delta s_{j, i}^{2}}},
$$

where $\Delta e_{j, i}$ and $\Delta s_{j, i}$ are the uncertainties related to the values of the observable $e_{j}$ and $s_{j}$ at points $i=1,2, \ldots, N_{j}$. We also define a level of agreement between experiments and simulations with respect to observable $j$ as

$$
R_{j}=\frac{\tanh \left[\left(d_{j}-d_{0}\right) / \lambda\right]+1}{2} .
$$

For $R_{j}=1$ theory and experiment give results that are well outside the respective error bars. For $R_{j}=0$ the results are within the error bars. A smooth transition between the two cases is provided by (3.3). Tests show that the conclusions of a validation exercise are not affected by the specific choices of parameters in the range $1 \leqslant d_{0} \leqslant 2$ and $0.1 \leqslant \lambda \leqslant 1$, and we believe that the values $d_{0}=1.5$ and $\lambda=0.5$ are reasonable choices.

The overall quality of the agreement between simulations and experiments can be evaluated by comparing different quantities at different hierarchy levels. To quantify such agreement, an appropriate metric should take into account all the errors associated with the model assumptions and the combinations of observables. To this aim, we have constructed a metric that casts the overall degree of agreement in the comparison into a single parameter. In this first attempt, the degree of agreement can be defined as the normalised sum over the considered set of observables of the product of three quantities:

$$
\chi=\frac{\sum_{j} R_{j} H_{j} S_{j}}{\sum_{j} H_{j} S_{j}}
$$

where $S_{j}$ indicates the sensitivity, or weight, attributed to observable $j$, and can be calculated from the relative error bars

$$
S_{j}=\exp \left(-\frac{\sum_{i} \Delta e_{j, i}+\sum_{i} \Delta s_{j, i}}{\sum_{i}\left|e_{j, i}\right|+\sum_{i}\left|s_{j, i}\right|}\right) .
$$

Naturally, the larger the error bars, the smaller the weight attributed to the comparison of that observable. $H_{j}$ quantifies the position of the variable in the primacy hierarchy. Although the definition of $H_{j}$ is somewhat arbitrary, we choose it to be a decreasing function of the hierarchy levels such as $H_{j}=1 / h_{j}$, where $h_{j}$ is the combined experimental/simulation primacy hierarchy level (see Ricci et al. (2009)) for a list of the hierarchy levels relative to LP measurements). For $H_{j}=1$, no assumptions or combinations of measurements are used for obtaining an observable. 
(a)

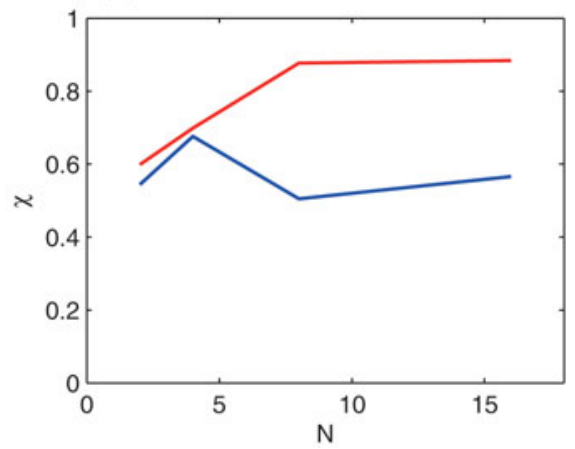

(b)

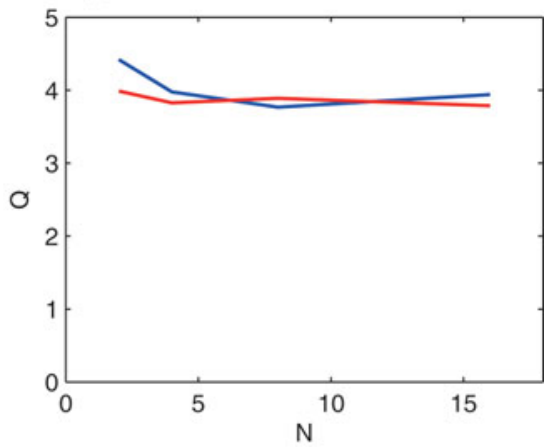

FIGURE 7. The results of the GBS validation against TORPEX experimental data are shown. The global metric, $\chi$, and the quality of the validation, $Q$, is shown for the three-dimensional GBS simulations (blue line) and the two-dimensional one (red line), as a function of $N$, the number of field line turns in the SMT.

Following this validation procedure, we compare experiments and simulations on the TORPEX device through a scan in the $N$ parameter, which allows investigating the transition from $k_{\|}=0$ mode dominated turbulence to $k_{\|} \neq 0$ turbulence (see Sec. 3.1). Using a set of observables, comprising data from a variety of electrostatic probes, we validate two models: a three-dimensional GBS simulations, able to describe the global evolution of TORPEX plasmas (Ricci and Rogers 2010), and the reduced twodimensional GBS simulations (Ricci et al. 2008), able to describe only the evolution of $k_{\|}=0$ modes. The results in Fig. 7 clearly show that the validation metric is able to capture the limit of the 2D model. In fact, the agreement of the $2 \mathrm{D}$ simulations and the experiment is no longer satisfactory when $k_{\|} \neq 0$ modes are present in the experiment, which occurs at $N \gtrsim 7$. We note that the trends of $Q$ and $\chi$ as a function of $N$ provide a synthetic and comprehensive way of representing the overall agreement between experiments and simulations. A deeper analysis can be performed by a looking at the validation table, as described in (Ricci and Rogers 2009; Ricci et al. 2011). This provides the level of agreement for each individual observable and it points out that GBS is able to well predict the modes that dominate the transport, while it overestimates the plasma pressure scale length. Possible GBS improvements that could lead to a better agreement between GBS results and experiments are discussed in Ricci et al. (2011). The use of GBS to model SOL physics can be found in Halpern et al. (2013), Loizu et al. (2013), Mosetto et al. (2013), Ricci and Rogers (2013), Halpern et al. (2014) and Loizu et al. (2014).

The proposed methodology can be easily applied to discriminate among models and to check how the agreement varies with specific control parameters. The dependence of $\chi$ upon these parameters may indicate the presence of a regime where a particular model is not appropriate. The most direct use of the proposed validation methodology is the determination of the most adequate code for a specific parameter regime. On the other hand, it is clear that it is much more difficult to judge a model in absolute terms.

\section{Future research}

In TORPEX, several advances have been achieved in the basic comprehension of waves, instabilities and turbulence in simple magnetized toroidal configurations. 


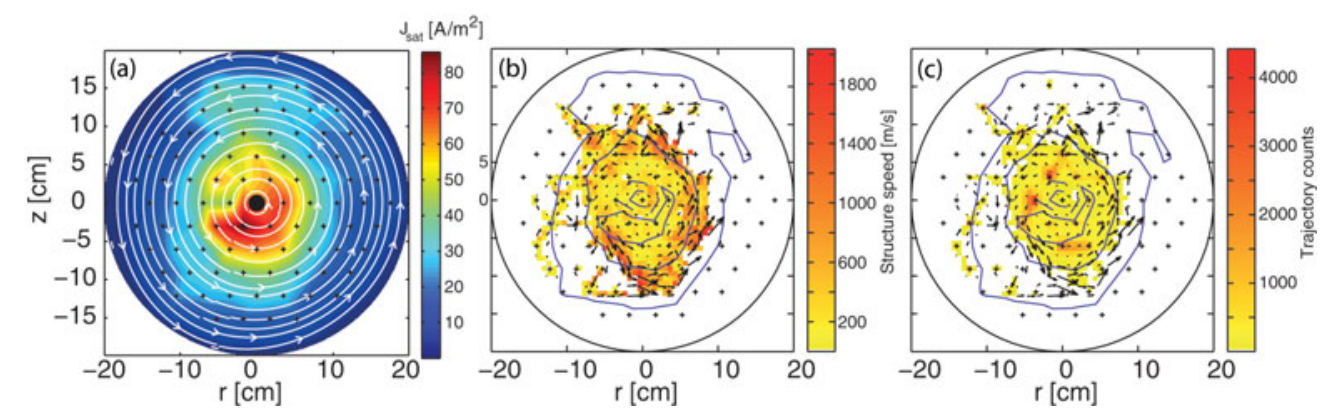

FIGURE 8. (a) Example of closed-field line geometry featuring quasi-circular flux surfaces. Also shown is the time-averaged profile of the ion saturation current density. From HEXTIP data: $2 D$ poloidal distribution of (b) structure trajectories, average velocity field and (c) number of structures detected. Contours of the time-averaged ion saturation current density profile are shown at $25 \%$ (outermost), $50 \%$ and $75 \%$ of the maximum.

The recently developed internal toroidal conductor system (see Sec. 2.2) opens new avenues for research of direct relevance for magnetically confined plasmas for fusion. An example of a closed-field line configuration with quasi-circular flux surfaces is shown in Fig. 8(a) with the time-averaged poloidal distribution of the ion saturation current density from HEXTIP data. This configuration is obtained with approximately 600 A flowing in the toroidal conductor and with the vertical magnetic field finely tuned to produce a quasi-circular magnetic geometry (Avino et al. 2014). Preliminary measurements of electrostatic fluctuations clearly indicate the presence of instabilities in the region of strong gradients, with a clear ballooning character, i.e. larger amplitudes on the LFS, in the region of unfavorable curvature (Fasoli et al. 2013). These fluctuations are associated with nonlinearly developed and saturated structures, as shown in Fig. 8(b) by the poloidal distribution of structure trajectories.

These more complex magnetic configurations will be the starting point of our future investigations to further advance our understanding of turbulence in the presence of closed/open field line regions. We intend to identify the instabilities from which turbulence is generated, to study their nonlinear development (wave-wave and wave-particle interactions) and saturation mechanisms, and the generation of macroscopic structures as was done for the SMT case. Different external control parameters will be sought to affect the fluctuation behavior, including the vertical magnetic field, the microwave power and the neutral gas pressure. Applying the same techniques on numerical data and experimental data allows testing the accuracy of these techniques, and provides a basis for a benchmark of the numerical simulation, which is necessary to determine the complexity of the numerical model needed for a realistic description not only of TORPEX data but especially for fusion devices.

On the theory side, starting from the simulations of the TORPEX device, the GBS code has been recently upgraded to describe more complex magnetic configurations. Future developments will include the implementation of a flexible numerical algorithm to describe more complex geometries, in particular the presence of an X point, and of a kinetic solver for the neutral atoms. TORPEX will continue to provide an ideal validation testbed for the future developments of GBS. 


\section{Acknowledgements}

The authors would like to thank the CRPP technical team for their essential support. Fruitful discussions with members of the team Superdiffusive Transport in Space Plasmas and its Influence on Energetic Particle Acceleration and Propagation at the ISSI are acknowledged. This work was supported in part by the Swiss National Science Foundation.

\section{REFERENCES}

Avino, F., Fasoli, A. and Furno, I. 2014 The new TORPEX in-vessel toroidal conductor for the generation of a poloidal magnetic field. Rev. Sci. Instrum. 85(3), 2014.

Bovet, A., Furno, I., Fasoli, A., Gustafson, K. and Ricci, P. 2012 Investigation of fast ion transport in TORPEX. Nuclear Fusion 52(9), 094017.

Bovet, A., Furno, I., Fasoli, A., Gustafson, K. and Ricci, P. 2013 Three-dimensional measurements of non-diffusive fast ion transport in TORPEX. Plasma Phys. Control. Fusion 55(12), 124021.

Bovet, A., Gamarino, M., Furno, I., Ricci, P., Fasoli, A., Gustafson, K., Newman, D. E. and Sanchez, R. 2014 Transport equation describing fractional Lévy motion of suprathermal ions in TORPEX. Nuclear Fusion 54(10), 104009.

Diallo, A., Fasoli, A., Furno, I., Labit, B., Podestà, M. and Theiler, C. 2008 Dynamics of plasma blobs in a shear flow. Phys. Rev. Lett. 101, 115005.

Diallo, A., Ricci, P., Fasoli, A., Furno, I., Labit, B., Müller, S. H., Podestà, M., Poli, F. M. and Skiff, F. 2007 Antenna excitation of drift wave in a toroidal plasma. Phys. Plasmas 14(10), 102101.

D’Ippolito, D. A., Myra, J. R. and Zweben, S. J. 2011 Convective transport by intermittent blobfilaments: comparison of theory and experiment. Phys. Plasmas 18(6), 060501.

Fasoli, A. et al. 2003 Electrostatic turbulence and transport in a simple magnetized plasma. Bull. Am. Phys. Soc. 13, 119.

Fasoli, A. et al. 2010 Electrostatic instabilities, turbulence and fast ion interactions in the TORPEX device. Plasma Phys. Control. Fusion 52(12), 124020.

Fasoli, A. et al. 2013 Basic investigations of electrostatic turbulence and its interaction with plasma and suprathermal ions in a simple magnetized toroidal plasma. Nuclear Fusion 53(6), 063013.

Federspiel, L., Labit, B., Ricci, P., Fasoli, A., Furno, I. and Theiler, C. 2009 Observation of a critical pressure gradient for the stabilization of interchange modes in simple magnetized toroidal plasmas. Phys. Plasmas 16(9), 092501.

Furno, I., Diallo, A., Fasoli, A., Labit, B., Müller, S. H., Plyushchev, G., Podestà, G. and M., Poli F. 2006 Effect of the magnetic configuration on fluctuations and turbulence in a magnetized toroidal plasma. In: Proc. of 11th EU-US Transport Task Force Meeting, http://www-fusionmagnetique.cea.fr/ttf2006/, Marseille, France.

Furno, I. et al. 2008a Mechanism for blob generation in the TORPEX toroidal plasmaa). Phys. Plasmas 15(5), 055903.

Furno, I., Spolaore, M., Theiler, C., Vianello, N., Cavazzana, R. and Fasoli, A. 2011a Direct twodimensional measurements of the field-aligned current associated with plasma blobs. Phys. Rev. Lett. 106, 245001.

Furno, I., Theiler, C., Chabloz, V., Fasoli, A. and Loizu, J. 2014 Pre-sheath density drop induced by ion-neutral friction along plasma blobs and implications for blob velocities. Phys. Plasmas 21(1), 012305.

Furno, I., Theiler, C., Lançon, D., Fasoli, A., Iraji, D., Ricci, P., Spolaore, M. and Vianello, N. 2011b Blob current structures in TORPEX plasmas: experimental measurements and numerical simulations. Plasma Phys. Control. Fusion 53(12), 124016.

Furno, I. et al. 2008b Experimental observation of the blob-generation mechanism from interchange waves in a plasma. Phys. Rev. Lett. 100(5), 055004.

Garcia, O. E. 2012 Stochastic modeling of intermittent scrape-off layer plasma fluctuations. Phys. Rev. Lett. 108, 265001.

Garcia, O. E., Naulin, V., Nielsen, A. H. and R., J. J. 2005 Turbulence and intermittent transport at the boundary of magnetized plasmas. Phys. Plasmas (1994-present) 12(6), 062309.

Goodman, T. P. et al. 2003 An overview of results from the TCV tokamak. Nuclear Fusion 43(12), 1619. 
Greenwald, M. 2010 Verification and validation for magnetic fusion. Phys. Plasmas 17(5), 058101.

Gustafson, K. and Ricci, P. 2012 Lévy walk description of suprathermal ion transport. Phys. Plasmas 19(3), 032304.

Gustafson, K., Ricci, P., Bovet, A., Furno, I. and Fasoli, A. 2012a Suprathermal ion transport in simple magnetized torus configurations. Phys. Plasmas 19(6), 062306.

Gustafson, K., Ricci, P., Furno, I. and Fasoli, A. 2012b Nondiffusive suprathermal ion transport in simple magnetized toroidal plasmas. Phys. Rev. Lett. 108, 035006.

Guszejnov, D., Lazanyi, N., Bencze, A. and Zoletnik, S. 2013 On the effect of intermittency of turbulence on the parabolic relation between skewness and kurtosis in magnetized plasmas. Phys. Plasmas 20(11), 112305.

Halpern, F. D., Ricci, P., Jolliet, S., Loizu, J. and Mosetto, A. 2014 Theory of the scrape-off layer width in inner-wall limited tokamak plasmas. Nuclear Fusion 54(4), 043003.

Halpern, F. D. et al. 2013 Theory-based scaling of the SOL width in circular limited tokamak plasmas. Nuclear Fusion 53(12), 122001.

Heidbrink, W. W. et al. 2012 Measurements of interactions between waves and energetic ions in basic plasma experiments. Plasma Phys. Control. Fusion 54(12), 124007.

Henderson, M. A. et al. 2003 Recent results from the electron cyclotron heated plasmas in tokamak à configuration variable (TCV). Phys. Plasmas 10(5), 1796-1902.

Iraji, D., Diallo, A., Fasoli, A., Furno, I. and Shibaev, S. 2008 Fast visible imaging of turbulent plasma in TORPEXa). Rev. Sci. Instrum. 79(10), 10F508.

Iraji, D., Furno, I. and Fasoli, A. 2011 Fast imaging of blob motion in TORPEX plasmas. IEEE Trans. Plasma Sci. 39(11), 3010-3011.

Iraji, D. et al. 2010 Imaging of turbulent structures and tomographic reconstruction of TORPEX plasma emissivity. Phys. Plasmas 17(17), 122304.

Katz, N., Egedal, J., Fox, W., Le, A. and Porkolab, M. 2008 Experiments on the propagation of plasma filaments. Phys. Rev. Lett. 101, 015003.

Krasheninnikov, S. I. 2001 On scrape off layer plasma transport. Phys. Lett. A 283(5-6), 368-70.

Krommes, J. A. 2008 The remarkable similarity between the scaling of kurtosis with squared skewness for TORPEX density fluctuations and sea-surface temperature fluctuations. Phys. Plasmas 15(3), 030703.

Labit, B. et al. 2007a Statistical properties of electrostatic turbulence in toroidal magnetized plasmas. Plasma Phys. Control. Fusion 49(12B), B281.

Labit, B., Furno, I., Fasoli, A., Diallo, A., Müller, S. H., Plyushchev, G., Podestà, M. and Poli, F. M. 2007b Universal statistical properties of drift-interchange turbulence in TORPEX plasmas. Phys. Rev. Lett. 98, 255002.

Labit, B., Theiler, C., Fasoli, A., Furno, I. and Ricci, P. 2011 Blob-induced toroidal momentum transport in simple magnetized plasmas. Phys. Plasmas 18(3), 032308.

Loizu, J. et al. 2013 On the electrostatic potential in the scrape-off layer of magnetic confinement devices. Plasma Phys. Control. Fusion 55(12), 124019.

Loizu, J., Ricci, P., Halpern, F. D., Jolliet, S. and Mosetto, A. 2014 Intrinsic toroidal rotation in the scrape-off layer of tokamaks. Phys. Plasmas 21(6), 062309.

Mekkaoui, A. 2013 Derivation of stochastic differential equations for scrape-off layer plasma fluctuations from experimentally measured statistics. Phys. Plasmas 20(1), 010701.

Mosetto, A., Halpern, F. D., Jolliet, S., Loizu, J. and Ricci, P. 2013 Turbulent regimes in the tokamak scrape-off layer. Phys. Plasmas 20(9), 092308.

Müller, S. 2007 Turbulence in basic toroidal plasmas. $P h D$ thesis, SB, Lausanne.

Müller, S. H., Fasoli, A., Labit, B., McGrath, M., Podestà, M. and Poli, F. M. 2004 Effects of a vertical magnetic field on particle confinement in a magnetized plasma torus. Phys. Rev. Lett. 93, 165003 .

Müller, S. H., Theiler, C., Fasoli, A., Furno, I., Labit, B., Tynan, G. R., Xu, M., Yan, Z. and Yu, J. H. 2009 Studies of blob formation, propagation and transport mechanisms in basic experimental plasmas (TORPEX and CSDX). Plasma Phys. Control. Fusion 51(5), 055020.

Müller, S. H. et al. 2006 Probabilistic analysis of turbulent structures from two-dimensional plasma imaging. Phys. Plasmas 13(10), 100701.

Müller, S. H. et al. 2008 Plasma blobs in a basic toroidal experiment: origin, dynamics, and induced transport. Phys. Plasmas 14, 110704. 
Myra, J. R. and D'Ippolito, D. A. 2005 Edge instability regimes with applications to blob transport and the quasicoherent mode. Phys. Plasmas 12(9), 092511.

Otsuji, K. et al. 2007 Small-scale magnetic-flux emergence observed with hinode solar optical telescope. Publ. Astron. Soc. Japan 59(sp3), S649-S654.

Park, J., Lühr, H., Stolle, C., Rother, M., Min, K. W. and Michaelis, I. 2010 Field-aligned current associated with low-latitude plasma blobs as observed by the champ satellite. Ann. Geophys. 28(3), 697-703.

Perri, S. and Zimbardo, G. 2012 Superdiffusive shock acceleration. Astrophys. J. 750(2), 87.

Perrone, D. et al. 2013 Nonclassical transport and particle-field coupling: from laboratory plasmas to the solar wind. Space Sci. Rev. 178(2-4), 233-270.

Piras, F. et al. 2009 Snowflake divertor plasmas on TCV. Plasma Phys. Control. Fusion 51(5), 055009 .

Plyushchev, G. et al. 2006 Fast ion source and detector for investigating the interaction of turbulence with suprathermal ions in a low temperature toroidal plasma. Rev. Sci. Instrum. 77(10), 10F503.

Podestà, M. 2007 Plasma production and transport in a simple magnetised toroidal plasma. $P h D$ thesis, SB, Lausanne.

Podestà, M., Diallo, A., Fasoli, A., Furno, I., Labit, B., Müller, S. H. and Poli, F. M. 2007 Characterization of the electron distribution function in an electron-cyclotron driven toroidal plasma. Plasma Phys. Control. Fusion 49(2), 175.

Podestà, M., Fasoli, A., Labit, B., Furno, I., Ricci, P., Poli, F. M., Diallo, A., Müller, S. H. and Theiler, C. 2008 Cross-field transport by instabilities and blobs in a magnetized toroidal plasma. Phys. Rev. Lett. 101, 045001.

Podestà, M., Fasoli, A., Labit, B., McGrath, M., Müller, S. H. and Poli, F. M. 2006 Experimental characterization and modelling of the particle source in an electron-cyclotron wave driven toroidal plasma. Plasma Phys. Control. Fusion 48(8), 1053.

Podestà, M. et al. 2005 Plasma production by low-field side injection of electron cyclotron waves in a simple magnetized torus. Plasma Phys. Control. Fusion 47(11), 1989-2002.

Poli, F. M., Brunner, S., Diallo, A., Fasoli, A., Furno, I., Labit, B., Müller, S. H., Plyushchev, G. and Podestà, M. 2006 Experimental characterization of drift-interchange instabilities in a simple toroidal plasma. Phys. Plasmas 13(10), 102104.

Poli, F. M., Podestà, M. and Fasoli, A. 2007 Development of electrostatic turbulence from driftinterchange instabilities in a toroidal plasma. Phys. Plasmas 14(5), 052311.

Poli, F. M., Ricci, P., Fasoli, A. and Podestà, M. 2008 Transition from drift to interchange instabilities in an open magnetic field line configuration. Phys. Plasmas 15(3), 032104.

Reames, D. V. 1999 Particle acceleration at the Sun and in the heliosphere. Space Sci. Rev. 90(3-4), 413-491.

Ricci, P., Halpern, F. D., Jolliet, S., Loizu, J., Mosetto, A., Fasoli, A., Furno, I. and Theiler, C. 2012 Simulation of plasma turbulence in scrape-off layer conditions: the gbs code, simulation results and code validation. Plasma Phys. Control. Fusion 54(12), 124047.

Ricci, P. and Rogers, B. N. 2009 Three-dimensional fluid simulations of a simple magnetized toroidal plasma. Phys. Plasmas 16(9), 092307.

Ricci, P. and Rogers, B. N. 2010 Turbulence phase space in simple magnetized toroidal plasmas. Phys. Rev. Lett. 104, 145001.

Ricci, P. and Rogers, B. N. 2013 Plasma turbulence in the scrape-off layer of tokamak devices. Phys. Plasmas 20(1), 010702.

Ricci, P., Rogers, B. N. and Brunner, S. 2008 High- and low-confinement modes in simple magnetized toroidal plasmas. Phys. Rev. Lett. 100, 225002.

Ricci, P., Theiler, C., Fasoli, A., Furno, I., Gustafson, K., Iraji, D. and Loizu, J. 2011 Methodology for turbulence code validation: quantification of simulation-experiment agreement and application to the TORPEX experiment. Phys. Plasmas 18(3), 032109.

Ricci, P., Theiler, C., Fasoli, A., Furno, I., Labit, B., Müller, S. H., Podestà, M. and Poli, F. M. 2009 Langmuir probe-based observables for plasma-turbulence code validation and application to the TORPEX basic plasma physics experiments. Phys. Plasmas 16(5), 055703.

Riva, F., Ricci, P., Halpern, F. D., Jolliet, S., Loizu, J. and Mosetto, A. 2014 Verification methodology for plasma simulations and application to a scrape-off layer turbulence code. Phys. Plasmas 21(6), 062301.

Ryutov, D. D. 2007 Geometrical properties of a snowflake divertor. Phys. Plasmas 14(6), 064502. 
Sandberg, I., Benkadda, S., Garbet, X., Ropokis, G., Hizanidis, K. and del-Castillo-Negrete, D. 2012 Universal probability distribution function for bursty transport in plasma turbulence. Phys. Rev. Lett. 103, 165001.

Schaffner, D. A., Carter, T. A., Rossi, G. D., Guice, D. S., Maggs, J. E., Vincena, S. and Friedman, B. 2012 Modification of turbulent transport with continuous variation of flow shear in the large plasma device. Phys. Rev. Lett. 109, 135002.

Terry, P. W. et al. 2008 Validation in fusion research: towards guidelines and best practices. Phys. Plasmas 15(6), 62503.

Theiler, C., Diallo, A., Fasoli, A., Furno, I., Labit, B., Podestà, M., Poli, F. M. and Ricci, P. 2008 The role of the density gradient on intermittent cross-field transport events in a simple magnetized toroidal plasma. Phys. Plasmas 15(4), 042303.

Theiler, C., Furno, I., Fasoli, A., Ricci, P., Labit, B. and Iraji, D. 2011a Blob motion and control in simple magnetized plasmasa. Phys. Plasmas 18(5), 055901.

Theiler, C., Furno, I., Kuenlin, A., Marmillod, Ph. and Fasoli, A. 2011b Practical solutions for reliable triple probe measurements in magnetized plasmas. Rev. Sci. Instrum. 82(1), 013504.

Theiler, C., Furno, I., Loizu, J. and Fasoli, A. 2012a Convective cells and blob control in a simple magnetized plasma. Phys. Rev. Lett. 108, 065005.

Theiler, C., Loizu, J., Furno, I., Fasoli, A. and Ricci, P. 2012b Properties of convective cells generated in magnetized toroidal plasmas. Phys. Plasmas 19(8), 082304.

Theiler, C. et al. 2009 Cross-field motion of plasma blobs in an open magnetic field line configuration. Phys. Rev. Lett. 103(6), 065001. 